\title{
Pacific
}

Journal of

Mathematics

\section{AN OVERDETERMINED PROBLEM IN POTENTIAL THEORY}

DMitry KHAVINSON, ERIK LUNDBERg AND RAZVAN TEODORESCU 


\title{
AN OVERDETERMINED PROBLEM IN POTENTIAL THEORY
}

\author{
DMitry KHAVINSON, ERIK LUndBERG ANd RAZVAN TEOdORESCU
}

\begin{abstract}
We investigate a problem posed by $L$. Hauswirth, F. Hélein, and F. Pacard (Pacific J. Math. 250:2 (2011), 319-334): characterize all the domains in the plane admitting a positive harmonic function that solves simultaneously the Dirichlet problem with null boundary data and the Neumann problem with constant boundary data. Hauswirth et al. suggested that essentially only three possibilities exist: the exterior of a disk, a half-plane, and a nontrivial example they found - the image of the strip $|\Im \zeta|<\pi / 2$ under $\zeta \mapsto \zeta+\sinh \zeta$. We partially prove their conjecture, showing that these are indeed the only possibilities if the domain is Smirnov and it is either simply connected or its complement is bounded and connected. We also show the nonexistence in $\mathbb{R}^{4}$ of an analogous nontrivial example among axially symmetric domains containing their axis of symmetry.
\end{abstract}

\section{Introduction}

In [Hauswirth et al. 2011], the authors posed the following problem: find a smooth bounded domain $\Omega$ in a Riemannian manifold $\mu_{g}$ with metric $g$, such that the first eigenvalue $\lambda_{1}$ of the Laplace-Beltrami operator on $\Omega$ has a corresponding real, positive eigenfunction $u_{1}$ satisfying $u_{1}=0, \partial u_{1} / \partial n=1$ on the boundary of $\Omega$. Any such domain is called extremal because it provides a critical point for the first eigenvalue $\lambda_{1}$ of the Laplace-Beltrami operator, under the constraint of fixed total volume of $\Omega$ (see [Hauswirth et al. 2011] and references therein).

In special cases, one can find a sequence of extremal domains $\left\{\Omega_{t}\right\}$ with increasing volumes, such that the limit domain $\Omega=\Omega_{t \rightarrow \infty}$ is unbounded, and its first eigenvalue vanishes as $t \rightarrow \infty$. This limit extremal domain is then called exceptional, and the corresponding limit function $\left(u_{1, t}\right)_{t \rightarrow \infty} \rightarrow u$ is a positive, harmonic function on $\Omega$ which solves simultaneously the overdetermined boundary value problem with null Dirichlet data and constant Neumann data.

The problem of finding exceptional domains in $\mathbb{R}^{n}$ and their corresponding functions $u$ (called roof functions by the authors of [Hauswirth et al. 2011]) is a

MSC2010: primary 31A25, 35R35, 35N25; secondary 30C20, 30E25.

Keywords: exceptional domain, roof function, vortex dynamics, quadrature domain, null-quadrature domains, non-Smirnov domain, Schwarz function, free boundary. 
nontrivial problem of potential theory. There is no obvious variational principle to use, on the one hand because $\Omega$ is unbounded (so the Dirichlet energy of $u$ [Astala et al. 2009, Chapter 1] will diverge), and, on the other hand, because the constant Neumann data constraint is not conformally invariant.

In the absence of a suitable variational formulation, we may interpret the scaling $t \rightarrow \infty$ described above as a dynamical process, in which the pair $\left(\Omega_{t}, u_{t}\right)$ evolves so that the limit $t \rightarrow \infty$ solves the overdetermined problem. In other words, we can turn this observation into a constructive method for finding (building) exceptional domains. In order to do this, it is helpful to note that, upon compactification of the boundary $\partial \Omega$ (with metric $d \sigma^{2}$ ), the pair $(\Omega, u$ ) with flat metric becomes conformal to the half-cylinder $\mathcal{N}:=\mathbb{R}_{+} \times \overline{\partial \Omega}$, with metric $d s^{2}=e^{-2 u}\left(d u^{2}+d \sigma^{2}\right)$. Under this reformulation, scaling of $\left(\Omega_{t}, u_{t}\right)_{t \rightarrow \infty}$ becomes equivalent to scaling of the metric structure given above, defined over the fixed space $\mathcal{N}$. This is reminiscent of the Ricci flow, in which the metric structure $g$ evolves with respect to a deformation parameter $t \in \mathbb{R}$ according to the equation

$$
\frac{d g_{i j}}{d t}=-2 R_{i j}
$$

with the right side of the equation given by the covariant Ricci tensor. It is known from [Topping 2006] that for the case of a two-dimensional manifold, with metric given by

$$
d s^{2}=e^{-2 u}\left(d x^{2}+d y^{2}\right),
$$

the Ricci flow equations reduce to a single nonlinear equation

$$
\frac{\partial u}{\partial t}=\nabla_{g}^{2} u
$$

(since in two dimensions the Riemann tensor has only one independent component). This is a heat equation with the generator given by the Laplace-Beltrami operator corresponding to the metric $d s^{2}$. Therefore, if there is a stationary solution $\partial u / \partial t \rightarrow$ 0 as $t \rightarrow \infty$, it will correspond to the scaling of the first eigenvalue $\lambda_{1}(t) \rightarrow 0$ and, by conformally mapping back $\mathcal{N}$ using the solution $u(t \rightarrow \infty)$, we will obtain the solution $(\Omega, u)$.

In other words, we can summarize this constructive method for finding exceptional domains in $\mathbb{R}^{2}$ as follows: starting from a 2-dimensional Riemannian manifold with finite volume and metric encoded through the positive real function $u$, and boundary set defined via $u=0$, consider the time evolution given by the Ricci flow, without volume renormalization. Then the manifold will remain Riemannian at all times [ibid.], and in the $t \rightarrow \infty$ limit, the function $u$ will become a solution of the nonlinear Laplace-Beltrami equation. Furthermore, if $u$ remains finite everywhere in the domain, then it is harmonic and satisfies both Dirichlet and Neumann conditions 
at all finite boundary components, so it is a solution for the overdetermined potential problem. Considered together with the (boundary) point at infinity, the manifold is equivalent to a pseudosphere - flat everywhere except at the point at infinity, with overall positive curvature. (We wish to emphasize that there is no reason to assume that such constructive methods would be exhaustive.)

Thus, so motivated, it is natural to try to characterize exceptional domains in flat Euclidean spaces. Hauswirth et al. [2011] suggested that in two dimensions there are only three examples: a complement of a disk, a half-plane, and a nontrivial example they found [ibid., Section 2]: the image of the strip $|\Im \zeta| \leq \pi / 2$ under the mapping $\zeta \mapsto \zeta+\sinh \zeta$. They posed as an open problem to determine if these are the only examples [ibid., Section 7]. (They gave some evidence by characterizing the half-plane under a global assumption on the gradient of the roof function [ibid., Proposition 6.1].) They also posed the problem of finding nontrivial examples in higher dimensions and suggested the possibility of axially symmetric examples similar to the nontrivial example in the plane [ibid., Remark 2.1].

We address both of these problems. The paper is organized as follows. In Section 2, we review the theory of Hardy spaces in order to address a subtlety that arises in connection with the regularity of the boundary of an exceptional domain. This leads us to assume in our theorems that the domain $\Omega$ is Smirnov. In Section 3, we characterize exteriors of disks as being the only exceptional domain whose boundary is compact. In Section 4, we establish a connection between the roof function of an exceptional domain and the so-called Schwarz function of its boundary, and we also show that the boundary of a simply connected exceptional domain $\Omega$ can pass either once or twice through infinity. We then show that in the first case $\Omega$ is a half-plane (Section 5) and in the second case $\Omega$ is the nontrivial example of Hauswirth et al. (Section 6). In each of these theorems we assume that $\Omega$ is Smirnov, but we allow the roof function to be a weak solution merely satisfying the boundary conditions almost everywhere.

In Section 7, we extend the result of Section 3 to higher dimensions. In Section 8, we show that the nontrivial example from Section 6 does not allow an extension to axially symmetric domains in four dimensions, contrary to what was suggested in [Hauswirth et al. 2011, Remark 2.1] (and we conjecture that this example has no analogues in any number of dimensions greater than two).

Sections 3 through 6 together partially confirm what was suggested in [Hauswirth et al. 2011, Section 7], under some assumptions on the topology of $\Omega$ and assuming that $\Omega$ is Smirnov. In Section 9, we give concluding remarks, including a conjecture that, up to similarity, there are only three finitely connected exceptional domains. The additional assumption of finite connectivity is due to a remarkable example of an infinitely connected exceptional domains that appeared in the fluid dynamics literature [Baker et al. 1976]. See Section 9 for discussion. 
Remark. After this paper was submitted, Martin Traizet [2013] announced a more complete classification of exceptional domains after developing an exciting new connection to minimal surfaces. He characterized the three examples as the only ones having finitely many boundary components. Traizet's preprint, which appeared while we were revising this paper, finds a new beautiful connection of the problem with the theory of minimal surfaces. From this point of view, he noticed the above-mentioned family of infinitely connected examples [Baker et al. 1976] and characterized them as the only periodic exceptional domains for which the quotient by the period has finitely many boundary components [Traizet 2013, Theorem 13]. For this latter result, he invokes a powerful theorem of W. H. Meeks and M. Wolf. Our methods mostly rely on classical function theory $\left(H^{p}\right.$ spaces) and potential theory and in most parts are different from Traizet's. Interestingly, as noted by Traizet [2013, Remark 5], if one could prove his Theorem 13 by only invoking pure function theory, this would give a new and independent proof of the Meeks-Wolf result from minimal surfaces. An attractive challenge!

\section{Classical versus weak solutions, regularity of the boundary, and Hardy spaces}

From the rigidity of the Cauchy problem, one might expect to obtain, for free, regularity of the boundary of an exceptional domain (as is often the case for solutions of free boundary problems). Unfortunately, the problem at hand is complicated by a remarkable family of examples with rectifiable but nonsmooth boundaries, also known as non-Smirnov domains; see [Duren 1970, Chapter 10]. This results in adding a Smirnov condition to the assumptions on the domains if we desire to consider weak solutions, i.e., harmonic roof functions satisfying the Dirichlet and Neumann boundary conditions almost everywhere with respect to the Lebesgue measure.

In order to address this subtlety, we first give some background from $H^{p}$ theory; see [Duren 1970] for details.

An analytic function $f: \mathbb{D} \rightarrow \mathbb{C}$ is said to belong to the Hardy class $H^{p}$, $0<p<\infty$, if the integrals

$$
\int_{0}^{2 \pi}\left|f\left(r e^{i \theta}\right)\right|^{p} d \theta
$$

remain bounded as $r \rightarrow 1$.

Recall that a Blaschke product is a function of the form

$$
B(z)=z^{m} \prod_{n} \frac{\left|a_{n}\right|}{a_{n}} \frac{a_{n}-z}{1-\bar{a}_{n} z},
$$

where $m$ is a nonnegative integer and $\sum\left(1-\left|a_{n}\right|\right)<\infty$. The latter condition ensures convergence of the product [Duren 1970, Theorem 2.4]. 
A function analytic in $\mathbb{D}$ is called an inner function if its modulus is bounded by 1 and its modulus has radial limit 1 almost everywhere on the boundary. If $S(z)$ is an inner function without zeros, then $S(z)$ is called a singular inner function.

An outer function for the class $H^{p}$ is a function of the form

$$
F(z)=e^{i \gamma} \exp \left\{\frac{1}{2 \pi} \int_{0}^{2 \pi} \frac{e^{i t}+z}{e^{i t}-z} \log \psi(t) d t\right\},
$$

where $\gamma$ is a real number, $\psi(t) \geq 0, \log \psi(t) \in L^{1}$, and $\psi(t) \in L^{p}$.

The following theorem [Duren 1970, Chapter 2, Chapter 5] (see also [Fisher 1983]) provides the parametrization of functions in Hardy classes by their zero sets, associated singular measures, and moduli of their boundary values.

Theorem 2.1. Every function $f(z)$ of class $H^{p}(p>0)$ has a unique (up to a unimodular constant factor) factorization of the form $f(z)=B(z) S(z) F(z)$, where $B(z)$ is a Blaschke product, $S(z)$ is a singular inner function, and $F(z)$ is an outer function for the class $H^{p}$.

Suppose $\Omega$ is a Jordan domain with rectifiable boundary and $f: \mathbb{D} \rightarrow \Omega$ is a conformal map. Then $f^{\prime} \in H^{1}$ by Theorem 3.12 in [Duren 1970]. By Theorem 2.1, $f^{\prime}$ has a canonical factorization $f^{\prime}(z)=B(z) S(z) F(z)$, and since $f$ is a conformal map $f^{\prime}$ does not vanish, so $f^{\prime}(z)=S(z) F(z)$. Then $\Omega$ is called a Smirnov domain if $S(z) \equiv 1$ so that $f^{\prime}(z)=F(z)$ is purely an outer function. This definition is independent of the choice of conformal map.

There are examples of non-Smirnov domains with, as above, $f^{\prime}(z)=S(z) F(z)$, but now $F(z) \equiv 1$ and the singular inner function $S(z)$ is not constant. Such examples were first constructed by M. Keldysh and M. Lavrentiev [1937] using complicated geometric arguments. Their existence was somewhat demystified by an analytic proof provided by P. Duren, H. S. Shapiro, and A. L. Shields [Duren et al. 1966; Shapiro 1966]. Like the disk, such a domain has harmonic measure at zero (assuming $f(0)=0$ ) proportional to arclength. Thus, its boundary is sometimes called a pseudocircle.

Similarly, there are exterior pseudocircles, arising as the boundary of an unbounded non-Smirnov domain [Jones and Smirnov 1999] for which the harmonic measure at infinity is proportional to arclength, and thus Green's function with singularity at infinity provides a roof function that is a weak solution satisfying the boundary conditions almost everywhere. Thus, this provides a pathological example of an exceptional domain in a weak sense. In order to construct such an unbounded non-Smirnov domain, let us follow the method above-mentioned [Duren et al. 1966], which is presented in Duren's book [1970, Section 10.4]. We recall that the construction is carried out by working backwards, first writing down a singular inner function $S(z)$ as a candidate for the derivative $f^{\prime}(z)$ of the conformal map $f(z)$. The difficulty is then to show that $f(z)$ is not only analytic, but is also 
univalent, so that it actually gives a conformal map from $\mathbb{D}$ to some domain $\Omega$. Univalence is established using a univalence criterion of Nehari (and Ahlfors and Weill), which states that the growth condition

$$
|(S f)(z)| \leq \frac{k}{\left(1-|z|^{2}\right)^{2}}, \quad \text { with } k<2,
$$

on the Schwarzian derivative $S f$ implies that $f$ maps $\mathbb{D}$ univalently onto a Jordan domain on the Riemann sphere.

We follow this procedure, indicating the step that needs to be modified. Start with a nondecreasing measure $\mu \leq 0$, singular with respect to Lebesgue measure on the circle, yet sufficiently smooth, so that it belongs to the Zygmund class $\Lambda_{*}$ [Duren 1970, Section 10.4].

We will also require $\mu$ to have first moment zero:

$$
\int_{0}^{2 \pi} e^{i \theta} d \mu(\theta)=0
$$

This can always be achieved by symmetrizing $\mu$ around the origin, thus replacing $\mu$ by $\frac{1}{2}(d \mu(\theta)+d \mu(\theta+\pi))$. Then the center of mass is at the origin, which is (2-3).

As in [Duren 1970, p. 177], let $F(z)$ be the Schwarz integral of $\mu$ :

$$
F(z)=\frac{1}{2 \pi} \int_{0}^{2 \pi} \frac{e^{i \theta}+z}{e^{i \theta}-z} d \mu(\theta) .
$$

Let $g(z)$ be the exponential of a constant (to be chosen later) times $F$ :

$$
g(z)=\exp \{-a F(z)\} .
$$

Having chosen $\mu$ in $\Lambda_{*}$ and nondecreasing, for $a$ small enough the antiderivative of $g$ maps the disk onto a bounded Jordan domain with rectifiable boundary [Duren 1970, Theorem 10.9]. Here is where we depart slightly from [Duren 1970], to get an unbounded domain as the image of $f(z)$. Instead of taking $g(z)$ as a candidate for $f^{\prime}(z)$, we take

$$
f^{\prime}(z)=g(z) / z^{2}
$$

and we check that the same estimates used in the proof of [Duren 1970, Theorem 10.9] also apply to this case. The residue of $f^{\prime}(z)$ is zero (since we made the first moment of $\mu$ zero), so its antiderivative $f(z)$ is analytic in $\mathbb{D}$ except for a simple pole at $z=0$. Also, $\left|f^{\prime}(z)\right|=1$ a.e. on $\partial \mathbb{D}$.

A calculation shows that the Schwarzian derivative $S f$ of $f$ is given by

$$
(S f)(z)=(S G)(z)+\frac{2}{z} \frac{g^{\prime}(z)}{g(z)}=-a F^{\prime \prime}(z)-\frac{a^{2}}{2} F^{\prime}(z)^{2}-\frac{2 a}{z} F^{\prime}(z),
$$

where $G$ is an antiderivative of $g$. As explicitly stated in [Duren 1970, Section 10.4], 
$F^{\prime \prime}(z), F^{\prime}(z)^{2}$, and $F^{\prime}(z)$ are each $O\left((1-|z|)^{-2}\right)$. Moreover, $F^{\prime}(0)=0$ by the vanishing of the first moment of $\mu$, so that $F^{\prime}(z) / z$ is also $O\left((1-|z|)^{-2}\right)$. Thus, if $a$ is chosen small enough, $S f$ satisfies the Nehari criterion for univalence, (2-2).

Hence, $f(z)$ is a conformal map taking $\{|z|<1\}$ onto the complement of a Jordan domain with rectifiable boundary. To see why the boundary is rectifiable, note that, as stated in [Duren 1970, Section 10.4]), $g(z) \in H^{1}$, and so $f^{\prime}(z)=g(z) / z^{2}$ is in $H^{1}$ in an annulus $0<r<|z|<1$.

This seemingly excessive construction of an exterior pseudocircle cannot be avoided by simply taking an inversion of an interior pseudocircle; the result will be non-Smirnov, but it will not be an exterior pseudocircle. Nor can one simply take the complement. As proved by P. Jones and S. Smirnov [1999], the complement of a non-Smirnov domain is often Smirnov! (This unexpected resolution of a long standing problem put to rest all hopes of characterizing the Smirnov property in terms of a boundary curve.)

Remark. Closely related examples of similar function-theoretic problems can be found in [Ebenfelt et al. 2002; Shahgholian 1992]. These examples of non-Smirnov exceptional domains lead to assuming $\Omega$ is Smirnov in our main theorems; but we allow $u$ to be a weak solution.

An alternative approach is to require the roof function $u$ to be a classical solution that satisfies the boundary condition everywhere (and not just a.e.). Then the domain must be Smirnov. This is because the boundary is locally real-analytic, as stated in the next lemma. Thus, the boundary is smooth except possibly at infinity. The preimage of infinity under a conformal map from the disc (or a circular domain, in the multiply connected case) can consist of at most countably many points on the boundary, i.e., infinity represents at most countably many "prime ends" [Markushevich 1977]. Indeed, the complement of $\bar{\Omega}$ is a disjoint union of domains, and for each boundary component of $\Omega$ going to infinity there is a separate component of the complement. None of these are void, since boundary components must be petals as opposed to slits - on a slit the normal vector would jump at the finite end point of the slit (violating the local real analyticity stated in the lemma below). There can be at most countably many such boundary components, because otherwise the complement of $\Omega$ would consists of uncountably many disjoint nonempty domains (impossible). Thus, the point at infinity can represent at most countably many prime ends (for the definition of prime end, see [Markushevich 1977, Chapter 2]). So, if the Smirnov condition is violated, meaning the derivative $f^{\prime}$ of the conformal map has a factor that is a nontrivial singular inner function, then the associated singular measure would consist of atoms. The derivative $f^{\prime}$ then decays exponentially at those points. This violates basic estimates on the derivatives of univalent functions, as given by Koebe's distortion theorem [Pommerenke 1975, Theorem 1.3]. 
Lemma 2.2. If $\Omega \subset \mathbb{R}^{2}$ is exceptional and the roof function $u$ is a classical solution in $C^{1}(\bar{\Omega})$, then $\partial \Omega$ is locally real-analytic.

Proof. The analytic completion $f(z)=u+i v$ (possibly multivalued) maps $\Omega$ into the right half-plane, since $u$ is positive. The Neumann condition for $u$ implies that $\left|f^{\prime}(z)\right|=1$ on $\partial \Omega$. Also, $u \in C^{1}(\bar{\Omega})$ implies that $f^{\prime} \in C(\bar{\Omega})$.

Choose a point $z_{0} \in \partial \Omega$, and let $\zeta_{0}=f\left(z_{0}\right)$. Let $g(\zeta)=f^{-1}(\zeta)$ denote the local inverse of $f(z)$. Choose a neighborhood $U$ of $\zeta$ and let $F:=\overline{U \cap\{\Re(\zeta) \geq 0\}}$. Choose $U$ small enough so that $g \in C(F)$.

Since $\left|g^{\prime}(\zeta)\right|=1$ on $\partial \Omega$, we can also choose $U$ small enough that $g^{\prime}$ does not vanish in $F$. This implies that $h(\zeta)=\log \left(g^{\prime}(\zeta)\right)$ is analytic in the interior of $F$ and continuous in $F$. Further, $\Re\{h(\zeta)\}$ vanishes on the imaginary axis, since $\left|g^{\prime}(\zeta)\right|=1$ there. Thus $h(\zeta)$ extends to a neighborhood of $\zeta_{0}$ by the Schwarz reflection principle. This allows us to extend $g^{\prime}(z)$, and therefore $g(z)$ and $f(z)$ extend analytically across $z_{0}$, since $u:=\Re f=0$ on $\partial \Omega$ and $|\nabla u|=1$ on $\partial \Omega$ near $z_{0}$.

Corollary 2.3. If $\partial \Omega$ is $C^{2}$-smooth and $\Omega$ is exceptional, then $\partial \Omega$ is locally realanalytic.

Proof. $C^{2}$-smoothness of $\partial \Omega$ implies that $u$ is in $C^{1}(\bar{\Omega})$. Now use Lemma 2.2.

Using Kellogg's theorem [1929] on regularity of conformal maps up to the boundary (see also [Pommerenke 1992, Chapter 3]), one easily extends the corollary to $C^{1, \alpha}$ boundaries $(\alpha>0)$ and even $C^{1}$ boundaries. We shall not pursue these details here. It would be interesting to find sharp necessary and sufficient conditions for the a priori regularity of the boundary that would guarantee the conclusion of Corollary 2.3. As we have mentioned in the beginning of this section, it is necessary to assume that the domain is Smirnov, but it is not at all obvious that this is sufficient. See [Castro and Khavinson 2013a; 2013b] for a related discussion regarding nonconstant functions in $E^{p}$ classes with real boundary values.

\section{The case when infinity is an isolated boundary point}

Theorem 3.1. Suppose $\Omega$ is an exceptional domain whose complement $\mathbb{C} \backslash \Omega$ is bounded and connected, and assume $\Omega$ is Smirnov. Then $\Omega$ is the exterior of a disk.

Proof. Let $u$ be a roof function for $\Omega$. Positivity of $u$ implies, by Bôcher's theorem [Axler et al. 1992, Chapter 3], $u(z)=u_{0}(z)+C \log |z|$ for some constant $C$, where $u_{0}(z)$ is harmonic in $\Omega \cup\{\infty\}$, and $u_{0}(z)$ approaches a constant at infinity (the Robin constant of $\partial \Omega$ provided $C=1)$. Thus, in view of the Dirichlet data of $u, u(z)$ is a multiple of the Green's function of $\Omega$ with a pole at infinity, and taking $v(z)$ to be the harmonic conjugate of $u(z) / C$, we have a conformal map $g(z)=e^{u(z) / C+i v(z)}$ from $\Omega$ to the exterior of the unit disk (note that $g(z)$ is single-valued in $\Omega$ ). 
Using both the Dirichlet and Neumann data, we have $\left|g^{\prime}(z)\right|=1 / C$ a.e. on $\partial \Omega$, and therefore

$$
\left|\left(g^{-1}\right)^{\prime}(\zeta)\right|=\frac{1}{\left|g^{\prime}\left(g^{-1}(\zeta)\right)\right|}=C
$$

a.e. on $\partial \mathbb{D}$. Since $g^{-1}$ has a simple pole at infinity, $\left(g^{-1}\right)^{\prime}$ is analytic. Also, $\left(g^{-1}\right)^{\prime}$ is in $H^{1}(\mathbb{C} \backslash \mathbb{D})$ since $\partial \Omega$ is rectifiable. Since $\Omega$ is Smirnov, the latter function is outer and also has constant modulus on the unit circle a.e., which together imply that it is constant. (Recall from (2-1) that an outer function is determined from its boundary values.) Hence, $g^{-1}$ is a linear function and $\partial \Omega$ is a circle.

We defer proving a higher-dimensional version of this result until Section 7, but we mention here that under more smoothness assumptions, the higher-dimensional case can be proved using a theorem of W. Reichel [1997].

Under additional smoothness assumptions, the hypothesis of Theorem 3.1 guarantees that $\Omega$ is a special type of arclength quadrature domain. The following is then an immediate corollary of a result of B. Gustafsson [1987, Remark 6.1].

Theorem 3.2. Suppose $\Omega$ is a finitely connected exceptional domain, with piecewise $C^{1}$ boundary, and infinity is not a point on the boundary of $\Omega$. Then $\Omega$ is the exterior of a disk.

This removes the condition that the complement of $\Omega$ is connected.

Proof. We will show that $\Omega$ is an arclength null-quadrature domain (this term is defined in Section 9.3) for analytic functions vanishing at infinity. At first, take the class of test functions to be integrated over $\partial \Omega$ to consist of rational functions $r(z)$ in $\Omega$ vanishing at infinity.

Let $f(z)=u(z)+i v(z)$ be the analytic completion of the roof function $u$. Note that $f^{\prime}(z)$ is single-valued (since it is the conjugate of the gradient of $u$ ), and, by the theorem of Bôcher already cited, $f^{\prime}(z)=O\left(|z|^{-1}\right)$. The inward normal of $\partial \Omega$ coincides with the gradient of $u$, which equals $\overline{f^{\prime}(z)}=1 / f^{\prime}(z)$. The unit tangent vector $d z / d s$ is a $90^{\circ}$ rotation of the normal vector $1 / f^{\prime}(z)$. Thus, $i f^{\prime}(z) d z=d s$. We then have a quadrature formula for integration of $r(z)$ with respect to arclength:

$$
\int_{\partial \Omega} r(z) d s=i \int_{\partial \Omega} r(z) f^{\prime}(z) d z=0,
$$

where the vanishing of this integral is obtained by deforming the contour to infinity where $f^{\prime}(z) r(z)=O\left(|z|^{-2}\right)$. Indeed, $r(z)=O\left(|z|^{-1}\right)$ by our choice of the test class, and $f^{\prime}(z)=O\left(|z|^{-1}\right)$ as mentioned above.

If the boundary of $\Omega$ is piecewise $C^{1}$, rational functions are dense in $E^{p}$ classes (see [Duren 1970, Theorem 10.7] and, for the multiply connected case, [Tumarkin and Havinson 1958a; 1958b; 1960]). In particular, rational functions $r(z)$ vanishing 
at infinity are dense in the space of functions $E(\Omega)$ considered in [Gustafsson 1987]. Thus, (3-1) shows that $\Omega$ is an arclength null-quadrature domain for this space of functions, and the result now follows from Remark 6.1 in [Gustafsson 1987].

\section{The Schwarz function of an exceptional domain}

The Schwarz function of a real-analytic curve $\Gamma$ is the (unique and guaranteed to exist near $\Gamma$ ) complex-analytic function that coincides with $\bar{z}$ on $\Gamma$. For the basics on the Schwarz function, we refer to [Davis 1974] and [Shapiro 1992].

We recall two basic facts needed in the proof of the next proposition.

(i) On $\Gamma,\left|S^{\prime}(z)\right|=1$.

(ii) The complex conjugate of $\sqrt{-S^{\prime}(z)}$ is normal to $\Gamma$.

Statement (i) follows from the chain rule and the fact that the complex conjugate of the Schwarz function, $\overline{S(z)}$, is an involution; see [Davis 1974, Chapter 7]. Statement (ii) follows from Formula (7.5) of the same reference, expressing the tangent vector along $\Gamma$ (i.e., the derivative of $z$ with respect to arclength):

$$
T(z)=d z / d s=1 / \sqrt{S^{\prime}(z)} .
$$

Proposition 4.1. If $\Omega$ is an exceptional domain such that the roof function is a classical solution, then the $z$-derivative of the roof function is given by $u_{z}(z)=c \sqrt{-S^{\prime}(z)}$, where $c$ is a real constant and $S(z)$ is the Schwarz function of $\partial \Omega$. In particular, $S^{\prime}(z)$ is analytic throughout $\Omega$.

Remark. If, for instance, the constant Neumann data for the roof function is 1, then the constant above $c= \pm \frac{1}{2}$, where the sign depends on the orientation of the boundary.

Proof. Lemma 2.2 implies that $\Gamma$ is locally real-analytic. So $\Gamma$ has a Schwarz function $S(z)$. The complex conjugate of the analytic function $u_{z}$ is normal to $\Gamma$ (since $u$ has zero Dirichlet data). In light of the constant Neumann data, we then have $\left|u_{z}(z)\right|=\left|\left(u_{z}(z)\right)^{*}\right|=\frac{1}{2}\left|u_{x}+i u_{y}\right|=\frac{1}{2} \sqrt{u_{x}^{2}+u_{y}^{2}}$ is constant on $\Gamma$. This, along with the statements (i) and (ii) above, shows that on $\Gamma$ the vectors $u_{z}(z)$ and $\sqrt{-S^{\prime}(z)}$ are parallel and each have constant length. Therefore, for some real constant $c$, the equation $u_{z}(z)=c \sqrt{-S^{\prime}(z)}$ holds on $\Gamma$. But since $u_{z}$ and $\sqrt{-S^{\prime}(z)}$ are both analytic, the equation is true everywhere that either side is defined. In particular, this guarantees analytic continuation of $S^{\prime}(z)$ throughout $\Omega$.

Let us use the Schwarz function to give a heuristic argument that the boundary of an exceptional domain can pass through infinity at most twice. In fact, the angle between consecutive arcs at infinity must be $\pi$ (and obviously there cannot be more than two such angles at infinity). Suppose the boundary of a domain has a corner where two arcs meet at an angle different from $0, \pi$, or $2 \pi$. Then the derivatives 
of the Schwarz functions of the two arcs have a branch cut along a third arc that propagates into the domain from the corner. To see why this is the case, note that the Schwarz function of an arc can be approximated near a point by the Schwarz function of the tangent line. Thus, to first order, the jump along the branch cut is linear, so to zeroth order, the jump of $S^{\prime}$ is determined by the slopes of the tangent lines. If the angle is 0 or $2 \pi$, then the tangent line is the same for each arc, but the orientation changes, so there is still a jump due to the sign change. In the case of an angle of $\pi$, both the tangent line and the orientation are unchanged. Thus, for any angle other than $\pi, S^{\prime}(z)$ has a jump across a branch cut between the two boundary components. For an exceptional domain, $u$ is a global solution throughout $\Omega$, and so Proposition 4.1 indicates that the Schwarz function cannot have such branch cuts. Thus, the angle between consecutive boundary arcs at infinity can only be $\pi$, and there can be at most two such angles.

In the informal argument above, we have assumed that each arc is real-analytic at infinity, so that the Schwarz function has a Taylor expansion there. A. Eremenko (private communication, 2012) related to us the following proof using ideas from [Barrett and Eremenko 2012] that extend techniques due to C. Pommerenke. Its indisputable advantage is that no regularity assumptions on $\partial \Omega$ are required. Also, an important part of the theorem readily extends to higher dimensions.

We recall that a Martin function is a positive harmonic function $M$ in a region $\Omega$ with the property that for any positive harmonic function $v$ in $\Omega$, the condition $v \leq M$ implies that $v=c M$, where $c>0$ is a constant. (Martin functions are also called minimal harmonic functions, as in [Heins 1950].) Martin functions on finitely connected domains are simply Poisson kernels evaluated at points of the Martin boundary, the boundary under Carathéodory compactification (prime ends) of the domain (see [Brelot 1971]).

Theorem 4.2 (A. Eremenko). The roof function $u$ of any exceptional domain $\Omega$ is a convex combination of at most two Martin functions of $\Omega$ at infinity. Moreover, $u(z)=O(|z|)$, and in two dimensions we also have $\nabla u(z)=O(1)$ in $\Omega$.

Remark. M. Traizet [2013] obtained the estimate $|\nabla u| \leq 1$ in $\Omega$ for domains with finitely many boundary components using the Phragmén-Lindelöf principle. For Smirnov domains $\Omega$, it suffices to show that $u_{z}$ belongs to the class $N^{+}$(see [Castro and Khavinson 2013a]) in order to conclude that the analytic function $u_{z}$ is bounded by 1 in $\Omega$. However, even this assumption is not needed here, and it is possible to establish the estimate on $\nabla u$ in full generality. Eremenko has kindly permitted us to include his argument here.

Proof. First we note that, as observed in [Barrett and Eremenko 2012, Lemma 1], if $u$ is a positive harmonic function in a disk (or a ball in higher dimensions), $D(a, R)$ 
of radius $R$ centered at $a$, and $u\left(z_{1}\right)=0$ for some boundary point $z_{1}$, then

$$
u(a) \leq 2 R\left|\nabla u\left(z_{1}\right)\right| .
$$

This immediately follows from Harnack's inequality for $D(a, R)$ as for $z \in D(a, R)$

$$
\frac{u(a)}{R+|z-a|} \leq \frac{u(z)}{R-|z-a|}=\frac{u(z)-u\left(z_{1}\right)}{R-|z-a|},
$$

and letting $z \rightarrow z_{1}$ establishes (4-2).

Applying (4-2) when $a \in \Omega$ and $R$ is the distance from $a$ to $\partial \Omega$, gives $u(a) \leq$ $2 R C \leq 2(|a|+$ const $) C$, where $C$ is the constant value of the Neumann data. So $u(z)=O(|z|)$, as $z \rightarrow \infty$, showing that $u(z)$ has order at most one.

That $u$ is a combination of at most two Martin functions now follows directly from [Kjellberg 1950, Theorem II], which states that if $\Omega$ has $n \geq 2$ different (nonproportional) Martin functions of respective orders $\rho_{1}, \rho_{2}, \ldots, \rho_{n}$, then

$$
\sum_{j=1}^{n} 1 / \rho_{j} \leq 2 .
$$

(This is proved using an application of Carleman's inequality [1926].) In higher dimensions, one must use [Friedland and Hayman 1976] instead of [Kjellberg 1950].

Next we show, in the two-dimensional case, the additional claim that $\nabla u(z)=$ $O(1)$. Let $R>0$ and consider an auxiliary function

$$
w_{R}=\frac{|\nabla u|}{u+R}
$$

where $R>0$ is a parameter. A direct computation shows that

$$
\Delta \log w_{R}=w_{R}^{2},
$$

and $w_{R}(z)=C / R$ for $z \in \partial D$. We claim that

$$
w_{R}(z) \leq 2 C / R \text { for } z \in \Omega,
$$

from which the result follows by letting $R \rightarrow \infty$, which gives $|\nabla u| \leq 2 C$ in $\Omega$.

Suppose, contrary to (4-4), that $w_{R}\left(z_{0}\right)>2 / R$ for some $z_{0} \in \Omega$. Here and in the next lines, we assume for simplicity that $C=1$. Let

$$
v(z)=\frac{2 R}{R^{2}-\left|z-z_{0}\right|^{2}} \quad \text { for } z \in D\left(z_{0}, R\right)=\left\{z:\left|z-z_{0}\right|<R\right\} .
$$

Obviously, $v(z) \geq 2 / R$. A computation reveals that $\Delta \log v=v^{2}$. Let

$$
K=\left\{z \in \Omega \cap D\left(z_{0}, R\right): w_{R}(z)>v(z)\right\} .
$$


We have $z_{0} \in K$, since $v\left(z_{0}\right)=2 / R$. Let $K_{0}$ be the component of $K$ containing $z_{0}$. Then we have $w_{R}(z)=v(z)$ on $\partial K_{0}$, since $w_{R}(z)<v(z)$ on $\partial \Omega \cap D\left(z_{0}, R\right)$ while $v(z)=+\infty$ on $\partial D\left(z_{0}, R\right)$. On the other hand,

$$
\Delta\left(\log w_{R}-\log v\right) \geq w_{R}^{2}-v^{2}>0 \quad \text { in } K_{0} .
$$

So the subharmonic function $\log w_{R}-\log v$ is positive in $K_{0}$ and vanishes on the boundary, a contradiction.

Remark. This a priori estimate implies the following corollary showing that the boundaries of exceptional domains are extremely regular. Namely, they are locally real-analytic and can even be parametrized from the unit circle via an antiderivative of a rational function. In particular, it validates the preceding argument using the Schwarz function, and establishes that the boundary passes at most twice through infinity each time with an angle of $\pi$. The only additional assumptions needed here are that the domain is Smirnov (compare Section 2) and simply connected.

Corollary 4.3. Let $\Omega$ be a simply connected Smirnov domain, and let $h(\zeta)$ be the conformal map from $\mathbb{D}$ to $\Omega$. If $\Omega$ is exceptional, then $h^{\prime}(\zeta)$ is a rational function, and we are in one of two cases:

(i) $h^{\prime}$ has one pole on $\partial \mathbb{D}$.

(ii) $h^{\prime}$ has two poles on $\partial \mathbb{D}$.

Proof. Let $u$ be a roof function for $\Omega$, and $f(z)=u+i v$ its analytic completion. Since $u>0, f(z)$ takes $\Omega$ into the right half-plane, and $f(h(\zeta))$ takes the unit disk $\mathbb{D}$ into the right half-plane. Adding an imaginary constant if necessary, we may assume that $f(h(0))>0$ is real. Then, by Herglotz's theorem [Hoffman 1962, Chapter 3; Duren 1970, Chapter 1], we can represent $f(h(\zeta))$ as

$$
f(h(\zeta))=\int_{\mathbb{T}} \frac{e^{i \theta}+z}{e^{i \theta}-z} d \mu(\theta),
$$

with $\mu$ positive.

Now, since $\Re f(h(\zeta))$ is the pullback to $\mathbb{D}$ of the roof function $u$, which by Theorem 4.2 is a convex combination of at most two Martin functions, $\mu$ consists of at most two atoms.

Thus, differentiating (4-5),

$$
f^{\prime}(h(\zeta)) \cdot h^{\prime}(\zeta)=R(\zeta),
$$

where $R(\zeta)$ is a rational function with either one or two double poles on $\partial \mathbb{D}$ (at the atoms of $\mu$ ). Since by Theorem 4.2, $f^{\prime}(h(\zeta))=2 u_{z}(h(\zeta)$ ) is a bounded analytic function in $\mathbb{D}$ with $\left|f^{\prime}(h(\zeta))\right|=1$ a.e. on $\partial \Omega, f^{\prime}(h(\zeta))$ is an inner function. Moreover, $h^{\prime}(\zeta)$ is an outer function, since $\Omega$ is Smirnov. 
For a rational function such as $R(\zeta)$, the canonical factorization given by Theorem 2.1 reduces to

$$
R(\zeta)=B(\zeta) \cdot F(\zeta)
$$

with $B$ a Blaschke product and $F$ a (rational) outer function. (The singular factor $S(\zeta)$ is trivial, since $R(\zeta)$ has no essential singularities.) By the uniqueness of the canonical factorization, $h^{\prime}(\zeta)$ and $f^{\prime}(h(\zeta)$ ) equal $F(\zeta)$ and $B(\zeta)$ respectively (up to multiplication by a unimodular constant). Hence, $h^{\prime}(\zeta)=F(\zeta)$ is rational, and $f^{\prime}(h(\zeta))=B(z)$ is a Blaschke product.

Remarks. (i) It is also possible to see that $f^{\prime}(h(\zeta))=B(z)$ has either no zeros or a single simple zero in $\mathbb{D}$. The reason is that the increment of the argument of $R^{\prime}(\zeta) d \zeta=d R$ over $\partial \Omega$ is zero, since $f^{\prime}(z) d z$ is positive. Thus, the winding number of $R^{\prime}(\zeta)$ over $\partial \Omega$ is negative one. If $R^{\prime}(\zeta)$ has one double pole on the boundary (recall that a pole on the boundary counts half [Bell 1992, p. 48]), then $R^{\prime}$ has no zeros inside $\mathbb{D}$. If $R^{\prime}(\zeta)$ has two double poles (each counts half), then $R^{\prime}(\zeta)$ has one simple zero.

(ii) It seems of interest to discuss to what extent the Corollary extends to the multiply connected case (Theorem 4.2 does not assume the domain is simply connected.) Let us make a few comments in this direction. Suppose $\Omega$ is a finitely connected Smirnov exceptional domain with the boundary passing through infinity and $n-1$ additional boundary components that are rectifiable Jordan curves. Let $z=h(w): K \rightarrow \Omega$ be a conformal map from a bounded circular domain into $\Omega$, with the outer circle $C_{n}$ mapped onto the unbounded component of $\partial \Omega$. Then it is possible to show that $u(h(\zeta))$ is a Poisson integral of a positive measure $\mu$ supported at one or two points on the circle $C_{n}$. Now $d u(h(\zeta)) / d \zeta=u_{z}(h(\zeta)) h^{\prime}(\zeta)$ is a single-valued analytic function in $K$ with at most two double poles at $\zeta_{1}, \zeta_{2}$, the support of $\mu$. Since the Poisson kernel $\partial_{n} g$ of $K$ is analytic in a neighborhood of $K$ except for $\zeta_{1}, \zeta_{2}$ on $C_{n}$, the function $g(\zeta):=d u(h(\zeta)) / d \zeta$ extends to a meromorphic function in a neighborhood of $C_{n} \backslash\left\{\zeta_{1}, \zeta_{2}\right\}$ and has poles at those points. (This gives us local real-analyticity of the contour going through $\infty$ on $\partial \Omega$.) Applying the argument principle as in the first part of this remark, we see that the total increment of the argument of $u_{z}(h(\zeta)) h^{\prime}(\zeta)$ is $n-2$, and $u_{z}(h(\zeta))$ has either $n$ or $n-1$ zeros in $K$. From an extension of the factorization theorem [Khavinson 1983] to the multiply connected case, it follows that $B(\zeta)=u_{z}(h(\zeta))$ is either a constant or a covering of the disk $\mathbb{D}$ with $n$ sheets. It is at most $n$ sheets by the above, and at least $n$ since each boundary component of $K$ is mapped to the circle and must have winding number at least one. Otherwise $B^{\prime}(\zeta)$ vanishes somewhere on the boundary of $K$, and a local expansion of $B^{\prime}$ at that point indicates that $B$ maps part of $K$ outside of $\mathbb{D}$, a contradiction. Putting all this together, either $h^{\prime}(\zeta)$ has two double poles 
or $B(\zeta)$ is constant. Thus, if $C_{n}$ passes through infinity only once, then $\Omega$ is a half-plane.

\section{Infinity as a single point on the boundary}

We now characterize the half-plane as the only simply connected exceptional domain having infinity as a single point on the boundary. This extends Proposition 6.1 of [Hauswirth et al. 2011] by removing a hypothesis $\left(\partial_{x} u>0\right.$ in $\left.\Omega\right)$ on the roof function.

Theorem 5.1. A domain $\Omega$ as in case (i) of Corollary 4.3 is a half-plane.

Remarks. (i) As mentioned in the introduction and Section 9, M. Traizet [2013] recently used minimal surfaces to establish this result under the assumption of finitely many boundary components. We note that, in the simply connected case, this assumption is stronger than ours, since infinitely many boundary components were allowed in Eremenko's result (Theorem 4.2). The final remark in the previous section explains how to use pure function theory in order to argue that $\Omega$ is a half-plane without the assumption that $\Omega$ is simply connected.

(ii) We have not been able to prove a higher-dimensional version of Theorem 5.1 (see also Section 7).

Proof. Using the same notation $f$ and $h$ from the proof of Corollary 4.3,

$$
f(h(\zeta))=\int_{\partial \mathbb{D}} \frac{e^{i \theta}+\zeta}{e^{i \theta}-\zeta} d \mu(\theta)
$$

for some finite positive measure $\mu$ on $\partial \mathbb{D}$. By assumption, we are in the case when $h^{\prime}$ has one pole, and according to the proof of Corollary 4.3, $\mu$ is an atomic measure with a single point mass. Without loss of generality, we can place it at the point $e^{i \theta}=1$.

Thus, $f(h(\zeta))=C \frac{1+\zeta}{1-\zeta}$, which upon differentiation gives

$$
f^{\prime}(h(\zeta)) h^{\prime}(\zeta)=2 C \frac{1}{(1-\zeta)^{2}}
$$

As asserted in the proof of Corollary 4.3, $f^{\prime}(h(\zeta))$ is the Blaschke factor of the right side, which has no zeros, so $f^{\prime}(h(\zeta))$ is a unimodular constant. Therefore, $f=u+i v$ is a linear function and $\Omega$ is a half-plane.

\section{Infinity as a double point of the boundary}

In this section we characterize the nontrivial example found in [Hauswirth et al. 2011]. Suppose $\Omega$ is a simply connected domain and $\Omega$ is exceptional. By Corollary 4.3, recall that the derivative $h^{\prime}(\zeta)$ of the conformal map from the disk onto $\Omega$ is a rational function with either one or two double poles on $\partial \mathbb{D}$. 
Theorem 6.1. A domain $\Omega$ as in case (ii) of Corollary 4.3 is, up to similarity, the image of the strip $|\Im w| \leq \pi / 2$ under the conformal map $g(w)=w+\sinh w$, while the analytic completion of the function $u(g(w))$ is the function $f(g(w))=\cosh w$.

Remark. Together with Theorems 3.1, 4.2 and 5.1, this shows that, under an assumption on the topology, the image of the strip under $\zeta \mapsto \zeta+\sinh \zeta$ is essentially the only nontrivial example of an exceptional domain in $\mathbb{R}^{2}$. The topological assumption is necessary, since there is a whole one-parameter family of nonsimilar infinitely connected exceptional domains (see Figure 3 on page 106). However, under the assumption of finitely many boundary components, the example described in Theorem 6.1 is the only nontrivial example, as recently proved in [Traizet 2013].

Proof. Using the same notation as in the proofs of Corollary 4.3 and Theorem 5.1, we have that $h^{\prime}(\zeta)$ is a rational function, and according to (4-6), $f^{\prime}(h(\zeta))$ is as well. This justifies applying the argument principle to study $f(h(\zeta))$ and $f^{\prime}(h(\zeta))$. Namely, we will prove the following.

Claim. The function $f$ solves the differential equation

$$
f^{\prime}=\sqrt{(f-1) /(f+1)}, \quad z \in \Omega,
$$

after simple normalizations described below.

Before proving this, we solve the differential equation to see that it gives the desired result. Separating variables,

$$
\int \sqrt{(f+1) /(f-1)} d f=z+C .
$$

Making the substitution $f=\cosh w$, we obtain $z=w+\sinh w$ (fixing the constant of integration $C=0)$. Now using the conditions $\Re f(z(w))=0$ for $z \in \partial \Omega$ and $\Re f(z(w))>0$ for $\zeta \in \Omega$, and the identity $\Re \cosh (x+i y)=\cosh x \cos y$, we find that the preimage of the domain in the $w$-plane is the strip $|\Im w| \leq \pi / 2$. Therefore, $\Omega$ can be described as the image of the strip under the map $z(w)=w+\sinh w$, concluding the proof of the theorem.

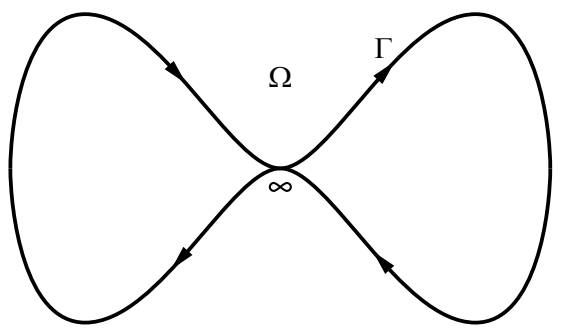

Figure 1. Local geometry of the boundary $\Gamma=\partial \Omega$ near infinity. 
To prove the claim, we will use the argument principle to show that both sides of (6-1) provide a conformal map from $\Omega$ to $\mathbb{D}$. From the formula

$$
T(z)=\frac{d z}{d s}=\frac{-i}{f^{\prime}(z)}=\frac{1}{\sqrt{S^{\prime}(z)}},
$$

which relates the tangent vector $T(z)$ on $\partial \Omega$ to the derivative of the analytic completion $f$ of $u(z)$, we obtain using the continuity of $T(z)$ through the double point at infinity (see Figure 1) that

$$
\oint_{\partial \Omega} d \log f^{\prime}(z)=2 \pi i
$$

We conclude that $f^{\prime}$ is a single-sheeted covering of the unit disk by the domain $\Omega$, and that it has only one zero, at some point $z_{0} \in \Omega$.

We may assume that $f\left(z_{0}\right)=1$. If not, say $f\left(z_{0}\right)=a+i b, a>0$, then one may subtract the constant $i b$ from $f$ (this just amounts to choosing a different harmonic conjugate for the same roof function), so we have $f\left(z_{0}\right)=a$. Then one may simply replace 1 with $a$ in the claim, and integrating the differential equation is done similarly resulting in a dilation of the original solution.

Now consider the function $g(z):=\sqrt{(f(z)-1) /(f(z)+1)}$, defined on $\Omega$ and taking values in the unit disk $\mathbb{D}$. This too is a univalent map from $\Omega$ into $\mathbb{D}$. Indeed, by the argument principle, $(f(z)-1) /(f(z)+1)$ is a branched, two-sheeted covering of the disk, since it maps each of the two boundary components shown in Figure 1 onto $\mathbb{T}$, Since the single branch point $z_{0}$ is mapped to the origin, taking the square root gives a single-valued analytic function.

Also, $f^{\prime}\left(z_{0}\right)=g\left(z_{0}\right)=0$. This uniquely determines the conformal map up to a unimodular constant, which we may assume is 1 (after a rotation), and we then arrive at the differential equation (6-1).

\section{An extension of Theorem 3.1 to higher dimensions}

In this section, we notice that some results in Section 3 extend to higher dimensions.

Theorem 7.1. Suppose $\Omega$ is an exceptional domain in $\mathbb{R}^{n}$ whose exterior is bounded and connected. If $\partial \Omega$ is $C^{2, \alpha}$-smooth, $\alpha>0$, then $\partial \Omega$ is a sphere.

Proof. Let $u$ be a roof function for $\Omega$, and let $v(s)=1 /|s|^{n-2}$ denote the Newtonian kernel. Fix $y \in \Omega$ and take a small ball $B_{\varepsilon}$ centered at $y$. Take also a large ball $B_{R}$ of radius $R$ that contains both $B_{\varepsilon}$ and the complement of $\Omega$.

Since $u(x)$ and $v(x-y)$ are harmonic in $\Omega \backslash B_{\varepsilon}$, Green's second identity gives

$$
\int_{\partial B_{R}+\partial \Omega-\partial B_{\varepsilon}}\left(v(x-y) \partial_{n} u(x)-u(x) \partial_{n} v(x-y)\right) d \sigma_{x}=0 .
$$


Letting $R \rightarrow \infty$, we can drop the integration over $\partial B_{R}$, since again by Bôcher's theorem [Axler et al. 1992, Chapter 3], near infinity $u(x) \approx|x|^{2-n}$.

Since, $u(x)=0$ on $\partial \Omega$ and $\partial_{n} u(x)=1$ on $\partial \Omega$,

$$
\int_{\partial \Omega} v(x-y) d \sigma_{x}=\int_{\partial B_{\varepsilon}}\left(v(x-y) \partial_{n} u(x)-u(x) \partial_{n} v(x-y)\right) d \sigma_{x} .
$$

Let $U$ be a bounded domain such that $\mathbb{R}^{n} \backslash \bar{U}=\Omega$. The outward normal for $\partial U$ is opposite to that of $\partial \Omega$, and since $v(x-y)=1 / \varepsilon^{n-2}$ on $\partial B_{\varepsilon}$,

$$
\int_{\partial U} v(x-y) d \sigma_{x}=\int_{\partial B_{\varepsilon}}\left(-\varepsilon^{-(n-2)} \partial_{n} u(x)+u(x) \partial_{n} v(x-y)\right) d \sigma_{x} .
$$

For the first term on the right, we have

$$
\int_{\partial B_{\varepsilon}} \varepsilon^{-(n-2)} \partial_{n} u(x) d \sigma_{x}=\int_{B_{\varepsilon}} \Delta u(x) d V=0 .
$$

So,

$$
\int_{\partial U} v(x-y) d \sigma_{x}=\int_{\partial B_{\varepsilon}} u(x) \partial_{n} v(x-y) d \sigma_{x} \rightarrow u(y),
$$

as $\varepsilon \rightarrow 0$. So, $u(y)$ is the single layer potential with charge density 1 on the surface $\partial U$. That $U$ is a ball now follows from Theorem 1 of [Reichel 1997].

Remark. Reichel's result holds for more general elliptic operators than the Laplacian. In the setting of the Laplacian, J. L. Lewis and A. Vogel [1992] characterized the sphere in terms of its interior Green's function under weaker regularity assumptions, namely, the boundary is assumed Lipschitz. In that case, the Neumann condition can be assumed to hold almost everywhere on the boundary. Thus, the hypothesis of Theorem 7.1 could be weakened by checking that the proof in [Lewis and Vogel 1992] works for the exterior case we are interested in. Yet, we have chosen an easier and more transparent path to apply Reichel's result directly, even though it requires a stronger regularity on the boundary.

\section{Nonexistence of a higher-dimensional analog of the $\cosh z$ example}

The authors in [Hauswirth et al. 2011] expressed a suspicion (see Remark 2.1 in [Hauswirth et al. 2011]) that there exist $n$-dimensional, rotationally symmetric examples similar to the two-dimensional example $\left\{(x, y) \in \mathbb{R}^{2}:|y|<\pi / 2+\cosh x\right\}$, which appeared in Section 6. We show that there does not exist an exceptional domain in $\mathbb{R}^{4}$ whose boundary is generated by rotation about the $x$-axis of the (two-dimensional) graph of an even function.

Theorem 8.1. There does not exist a rotationally symmetric exceptional domain $\Omega$ in $\mathbb{R}^{4}$ that contains its own axis of symmetry and whose boundary is obtained by 
rotating the (two-dimensional) graph of an even real-analytic function about the $x$-axis.

Our proof will rely heavily on two tricks, one exploiting the assumption that $n=4$, and the other using the assumption that the generating curve is symmetric. However, we strongly suspect a more general nonexistence of such examples in $\mathbb{R}^{n}$ for any $n>2$.

Therefore, we conjecture the following.

Conjecture. For $n>2$, there does not exist an axially symmetric, exceptional domain in $\mathbb{R}^{n}$ that contains its own axis of symmetry.

Remark. The assumption that the domain contains its axis of symmetry rules out the exteriors of balls and circular (or spherical) cylinders, respectively (which are clearly exceptional domains as was noted in [Hauswirth et al. 2011]). Also, A. Petrosyan and K. Ramachandran pointed out to us (private communication, 2012) that the nonconvex component of the exterior of a certain cone is also an exceptional domain. In $\mathbb{R}^{4}$, using the $x$-axis as the axis of rotation, the cone is the rotation of $\left\{(x, y): y^{2}-x^{2}=0\right\}$, and the roof function in the meridian coordinates $x, y$ where $y$ is the distance to the $x$-axis in $\mathbb{R}^{4}$, is $u(x, y)=\left(y^{2}-x^{2}\right) / y$ for $y>0$.

Proof of Theorem 8.1. Suppose that $\Omega$ is such a domain in $\mathbb{R}^{4}$. Namely, the boundary $\partial \Omega$ is obtained from rotation of $\gamma:=\left\{(x, y) \in \mathbb{R}^{2}: y=g(x)\right\}$, with $g(-x)=g(x)$. That is, the boundary of $\Omega$ is given by

$$
\left\{\left(x_{1}, x_{2}, x_{3}, x_{4}\right) \in \mathbb{R}^{4}: \sqrt{x_{2}^{2}+x_{3}^{2}+x_{4}^{2}}=g\left(x_{1}\right)\right\} .
$$

Considering the boundary data, the rotational symmetry of the domain will be passed to the roof function (this requires uniqueness of the roof function guaranteed by the Cauchy-Kovalevskaya theorem, which may be applied since the boundary is assumed to be real-analytic), so that, abusing notation, we can write

$$
u\left(x_{1}, x_{2}, x_{3}, x_{4}\right)=u(x, y) .
$$

For clarity, we emphasize that the $x$-axis corresponds to the axis of symmetry and the $y$-coordinate gives the distance from the axis of symmetry.

For axially symmetric potentials $v$ in $\mathbb{R}^{n}$, the cylindrical reduction of Laplace's equation is

$$
\Delta_{(x, y)} v+(n-2) v_{y} / y=0,
$$

where $x=x_{1}$ and $y=\sqrt{x_{2}^{2}+\cdots+x_{n}^{2}}$. Moreover, in the case we are considering, when $n=4, u$ satisfies the equation $\Delta u+\left(2 u_{y}\right) / y=0$, if and only if $y u(x, y)$ is a harmonic function of two variables $x$ and $y$. Indeed,

$$
\Delta(y u)=y \Delta u+2 \nabla u \cdot \nabla y+u \Delta y=y \Delta u+2 u_{y} .
$$


(The trick that reduces axially symmetric potentials in $\mathbb{R}^{4}$ to harmonic functions in the meridian plane is well known: compare [Khavinson 1991; Karp 1992].)

Since $y u(x, y)$ is then harmonic in the unbounded two-dimensional domain $D$ bounded by $\gamma$ and its reflection (which we denote by $\bar{\gamma}$ ) with respect to the $x$-axis, this implies $\partial(y u(x, y)) / \partial z$ is analytic in the domain $D$, where as usual $z=x+i y$. The Cauchy data (originally posed in $\left.\mathbb{R}^{4}\right)$ imply that $u_{z}=\frac{1}{2}\left(u_{x}-i u_{y}\right)$ coincides with $\sqrt{-S^{\prime}(z)}$ on $\gamma$ and $\bar{\gamma}$. This implies that the analytic function

$$
W(z):=(y u)_{z}=(-i / 2) u+y u_{z}
$$

coincides with $((z-S(z)) / 2 i) \sqrt{-S^{\prime}(z)}$ on $\gamma$ and $\bar{\gamma}$. The latter function is analytic, so this actually gives a formula for $W(z)$ whose validity is not limited to $\gamma$ and $\bar{\gamma}$ :

$$
W(z)=\frac{z-S(z)}{2 i} \sqrt{-S^{\prime}(z)} .
$$

We note that (8-2) can be used to analytically continue $S(z)$ to all of $D$, but this is not needed in our proof.

Let $f(\zeta)$ be the conformal map from the strip $\Sigma:=\left\{|\Im \zeta|<\frac{1}{2}\right\}$ to $D$ such that $f(0)=0$ and $\arg \left\{f^{\prime}(0)\right\}=0$. The two-fold symmetry of $D$ implies that $f(\zeta)$ is an odd function. Indeed, otherwise $h(\zeta)=-f(-\zeta)$ gives another conformal map from the strip $\Sigma$ to $D$. But, $h(0)=-f(0)=0$ and $h^{\prime}(0)=f^{\prime}(0)$ implies $h=f$, by the uniqueness of the conformal map (up to choice of $f(0)$ and argument of $f^{\prime}(0)$ ).

The Schwarz functions $S_{t}, S_{b}$ of the top and bottom edges of the strip $\Sigma$ are $S_{t}(\zeta)=\zeta-i$ and $S_{b}(\zeta)=\zeta+i$. In terms of the conformal map $f(\zeta)$, the pullbacks to the $\zeta$-plane of the Schwarz functions $S_{+}$and $S_{-}$of $\gamma$ and $\bar{\gamma}$, respectively, satisfy (see [Davis 1974, Chapter 8, Equation 8.7])

$$
S_{ \pm}(f(\zeta))=f(\zeta \mp i) \quad \text { and } \quad S_{ \pm}^{\prime}(f(\zeta))=\frac{f^{\prime}(\zeta \mp i)}{f^{\prime}(\zeta)}
$$

Substituting these into (8-2), we obtain two expressions for the pullback of $W(z)$ to the strip $\Sigma$ :

$$
\frac{f(\zeta)-f(\zeta \mp i)}{2 i} \sqrt{-\frac{f^{\prime}(\zeta \mp i)}{f^{\prime}(\zeta)}}
$$

Even though $W(f(\zeta))$ is analytic throughout $\Sigma$, we caution that these two expressions (one expression for + and one for - ) may only be valid near the bottom and top sides (respectively) of the strip $\Sigma$.

Claim. The function $W(f(\zeta))$ is odd.

In view of the claim, $W(0)=W(f(0))=0$. By (8-1), we then have that $(-i / 2) u+y u_{z}=0$ at $z=0$, which implies that $u(0,0)=0$. This contradicts the positivity of $u$, proving the theorem. 
There remains to prove the claim. Set $V(\zeta)=W(f(\zeta))+W(f(-\zeta))$. Using (8-3), we can write

$$
V(\zeta)=\frac{f(\zeta)-f(\zeta-i)}{2 i} \sqrt{-\frac{f^{\prime}(\zeta-i)}{f^{\prime}(\zeta)}}+\frac{f(-\zeta)-f(-\zeta+i)}{2 i} \sqrt{-\frac{f^{\prime}(-\zeta+i)}{f^{\prime}(-\zeta)}}
$$

We show that this formula vanishes where it is valid, which then implies that $V(\zeta)$ vanishes identically throughout $\Sigma$. For this, we use the fact that $f$ is odd and consequently $f^{\prime}$ is even.

$$
V(\zeta)=\frac{f(\zeta)-f(\zeta-i)}{2 i} \sqrt{-\frac{f^{\prime}(\zeta-i)}{f^{\prime}(\zeta)}}+\frac{-f(\zeta)+f(\zeta-i)}{2 i} \sqrt{-\frac{f^{\prime}(\zeta-i)}{f^{\prime}(\zeta)}}=0
$$

This establishes the claim.

\section{Concluding remarks and main conjecture}

9.1. It is tempting to conjecture that the three examples in the plane studied above are the only exceptional domains in the plane, as suggested in [Hauswirth et al. 2011]. However, there is a remarkable family of infinitely connected exceptional domains. They were discovered as solutions to a fluid dynamics problem by Baker, Saffman, and Sheffield [Baker et al. 1976]. (See also [Crowdy and Green 2011] for a more detailed account.) The original problem there was to find hollow vortex equilibria with an infinite periodic array of vortices, known as spinning bubbles, amid a stationary flow of ideal fluid. The domain occupied by fluid turns out to be an exceptional domain with an infinite periodic array of holes, and the roof function is a stream function of the fluid flow; see Figure 2. The constant Dirichlet condition corresponds to the requirement that the boundary of each hollow vortex is a stream line, and the constant Neumann condition corresponds to the requirement that the fluid pressure should be balanced at the interface by the pressure inside each bubble which is assumed constant. The latter correspondence is more subtle; in order to have constant pressure along a stream line, the fluid velocity (which equals the normal derivative of stream function) should be constant, by Bernoulli's law.

This infinitely connected example leads us to add to the conjecture the assumption that the domain is finitely connected.

Conjecture. The only finitely connected exceptional domains in $\mathbb{R}^{2}$ are the exterior of the unit disk, the half-plane, and the domain described in Theorem 6.1.

Remark. As mentioned in the introduction, Martin Traizet [2013] recently announced a classification of exceptional domains. His results confirm our conjecture for domains having finitely many boundary components and also show that the above infinitely connected example is the only periodic exceptional domain for which the quotient by the period has finitely many boundary components. His methods 


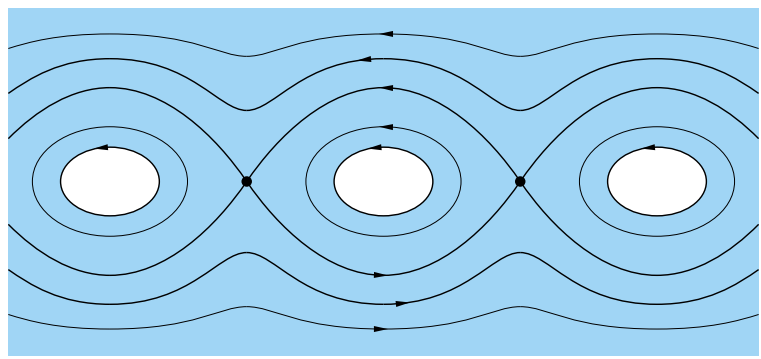

Figure 2. An infinitely connected exceptional domain that also provides a hollow vortex equilibrium. Level curves of the roof function are stream lines. The shape of the bubbles ensures that the pressure dictated by Bernoulli's law is constant at the fluid-bubble interface.

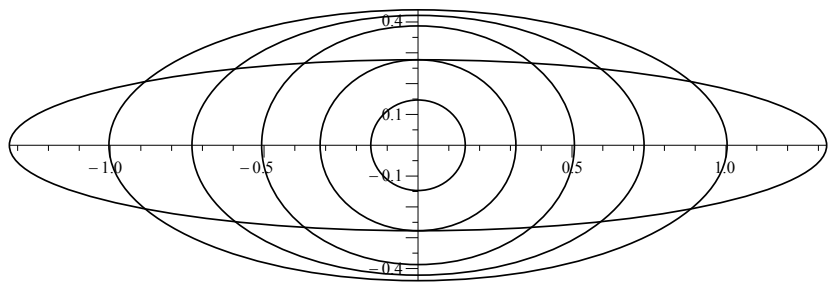

Figure 3. One-parameter family of bubble shapes associated with exceptional domains. As stated in [Traizet 2013], each of the three previously known examples can be recovered as scaling limits of this family. In that sense, this family includes all known examples.

use a remarkable nontrivial correspondence to minimal surfaces, perturbing an exceptional domain by harmonically mapping it to another domain in such a way that the graph of the new height function (which pulls back to the roof function in the original domain) satisfies the minimal surface equation. A miraculous (and crucial to his approach) by-product is that, whereas the graph of the roof function meets its boundary at a $45^{\circ}$ angle, the minimal graph meets its boundary vertically so that gluing it to its own reflection over the $x y$-plane results in a smooth minimal surface (without boundary!) embedded in $\mathbb{R}^{3}$.

9.2. Regarding the higher-dimensional case, we conjecture the following extension of Theorem 5.1 to higher dimensions.

Conjecture. Suppose $\Omega$ is an exceptional domain in $\mathbb{R}^{n}$ that is homeomorphic to a half-space. Then $\Omega$ is a half-space. 
9.3. The connection to the Schwarz function in Section 4 reveals that exceptional domains are arclength null-quadrature domains. That is, for any function $f$, say analytic in $\Omega$, continuous in $\bar{\Omega}$, integrable over the boundary, and decaying sufficiently at infinity, we have $\int_{\partial \Omega} f d s=0$. Indeed, $\int_{\partial \Omega} f d s=\int_{\partial \Omega} f(z)(1 / T(z)) T(z) d s=$ $\int_{\partial \Omega} f(z) \sqrt{S^{\prime}(z)} d z$, where $T(z)$ is the complex unit tangent vector (see Section 4), and now this integral vanishes as long as the integrand decays sufficiently at infinity. Null-quadrature domains were previously studied in the case of area measure. They were characterized in the plane by M. Sakai [1981]. Our current study can be seen as a step toward characterizing null-quadrature domains for arclength.

9.4. Other interesting connections involve differentials on Riemann surfaces. The study of Gustafsson [1987] used half-order differentials on the Schottky double of an arclength quadrature domain. From a different point of view, the boundary of an exceptional domain is a trajectory of the positive quadratic differential $-(d f)^{2}$, where $f(z)$ is the analytic completion of the roof function.

9.5. The differential equation (6-1) can be solved by a more general substitution using Jacobi elliptic functions [Abramowitz and Stegun 1964, p. 567, §16]:

$$
\begin{aligned}
f(\zeta, k) & =\cos \theta \operatorname{cn}(\zeta, k)+\sin \theta \operatorname{sn}(\zeta, k), \\
z(\zeta) & =\cos \theta \operatorname{sn}(\zeta, k)-\sin \theta \operatorname{cn}(\zeta, k)+\int^{\zeta} \operatorname{dn}(\xi, k) d \xi,
\end{aligned}
$$

where $\theta$ is an arbitrary phase, $\theta \in[0,2 \pi]$.

For a given value of the elliptic modulus $k \in[0,1]$, we define the corresponding domain $\mathbb{F}$ through its fundamental periods

$$
T_{1}(k)=4 K\left(\sqrt{1-k^{2}}\right) \quad \text { and } \quad T_{2}(k)=4 K(k),
$$

where

$$
K(k) \equiv \int_{0}^{\pi / 2} \frac{1}{\sqrt{1-k^{2} \sin ^{2} \theta}} d \theta
$$

is the complete elliptic integral of the first kind [Abramowitz and Stegun 1964, p. 590, §17.3]. It diverges for $k=1$ and equals $\pi / 2$ for $k=0$.

Then it is straightforward to check that (6-1) is satisfied by $f(z)$, due to the identity [Abramowitz and Stegun 1964, p. 573, §16.9]

$$
1=(-\operatorname{sn} z \cos \theta+\mathrm{cn} z \sin \theta)^{2}+(\operatorname{cn} z \cos \theta+\operatorname{sn} z \sin \theta)^{2} .
$$

Let $\gamma$ be the preimage of $\partial \Omega$ under $z(\zeta)$ : it consists of two pieces $\gamma_{ \pm}, \gamma_{-}=-\gamma_{+}$, dividing the fundamental domain $\mathbb{E}$ into three subdomains. Denote the component which contains the origin by $D_{0}$. Since $f(0)=1$, we conclude that $\Re f(z)>0$ for $z \in D_{0} \backslash \gamma_{ \pm}$, and we have proven the following result. 
Proposition. The exceptional domain $\Omega$ is the image of the domain $D_{0}(k)$ under the map $\zeta \mapsto z(\zeta)$ defined in (9-2).

Remark. The case discussed in the proof of the theorem corresponds to the degenerate elliptic modulus $k=0$. Then the domain $\mathbb{F}$ becomes the infinite strip

$$
T_{1}(0)=4 K(1) \rightarrow \infty, \quad T_{2}(0)=4 K(0)=2 \pi,
$$

while the functions $f, g$ become (using the fact that $\operatorname{dn}(z, 1) \equiv 1$ )

$$
z(\zeta)=\zeta+\sinh \zeta, \quad f(z(\zeta))=\cosh \zeta
$$

As noted before, the conditions $\left.\Re f(\zeta)\right|_{\gamma_{ \pm}}=0$ give the preimage $\gamma_{ \pm}:=z^{-1}(\partial \Omega)=$ $\{\Im \zeta= \pm \pi / 2\}$, and the preimage of the domain, $D_{0}$, becomes the strip $|\Im \zeta| \leq \pi / 2$.

9.6. The domain $D_{0}(k)$ is the preimage of the unit disk under the map $\zeta: \mathbb{F} \rightarrow \mathbb{D}$ defined by

$$
\zeta(w)=\frac{\operatorname{sn}(w, k)-i}{\operatorname{sn}(w, k)+i}, \quad k \in[0,1],
$$

with the support of $\mu$ at points $\zeta_{ \pm}= \pm(1-i k) /(1+i k)$, where $\mu$ is the measure discussed in the proof of Corollary 4.3. The case $k \rightarrow 0$ corresponds to the strip domain and to $\zeta_{ \pm}= \pm 1$. The reparametrization invariance noted above for the solution $f(z)$ of (6-1) under rescaling of the elliptic modulus $k$ is indicative of a deeper invariance of the solution: all the specific solutions in $\mathbb{C}$ discussed here are associated with fixed points in the moduli space of Riemann surfaces.

Again let $f(h(z))$ be the analytic completion of a solution, and denote by $\mathscr{G}$ the group of transformations that leaves $\operatorname{supp}(\mu)$ invariant up to a global rotation. It follows that $f$ is an automorphism of the quotient of the group of linear fractional transformations by $\mathscr{G}$, which can be in general a Kleinian group.. The limit set (accumulation points of the orbits of the group) can be finite (in which case it can consist of only 0,1 , or 2 points), or infinite. It is known (see [Astala et al. 2009, Theorem 10.3.4]) that the set of homeomorphic solutions for a quasilinear elliptic equation of Laplace-Beltrami type forms a group only in the case of finite limit set. The Kleinian groups are called degenerate in this case, and they correspond to either finite groups (with empty limit set), or cyclic groups (generated by one element, with limit set consisting of 1 or 2 points). These correspond to the solutions described in the present paper (isolated point at infinity, respectively simple and double boundary point at infinity).

\section{Acknowledgements}

The authors are indebted to Dimiter Vassilev for bringing the article [Hauswirth et al. 2011] to their attention. We wish to thank Alexandre Eremenko for sharing an 
improved proof of Theorem 4.2 and Arshak Petrosyan and Koushik Ramachandran for pointing out the example of a cone as an exceptional domain. We also wish to thank Martin Traizet for helpful discussion regarding his preprint. We are grateful to the anonymous referee whose careful reading of the paper and constructive criticism signifantly improved the clarity of the exposition. Khavinson and Lundberg acknowledge partial support from the NSF under the grant DMS-0855597.

\section{References}

[Abramowitz and Stegun 1964] M. Abramowitz and I. A. Stegun (editors), Handbook of mathematical functions with formulas, graphs, and mathematical tables, National Bureau of Standards Applied Mathematics Series 55, U.S. Government Printing Office, Washington, DC, 1964. Reprinted by Dover, New York, 1974. MR 29 \#4914 Zbl 0171.38503

[Astala et al. 2009] K. Astala, T. Iwaniec, and G. Martin, Elliptic partial differential equations and quasiconformal mappings in the plane, Princeton Mathematical Series 48, Princeton University Press, 2009. MR 2010j:30040 Zbl 1182.30001

[Axler et al. 1992] S. Axler, P. Bourdon, and W. Ramey, Harmonic function theory, Graduate Texts in Mathematics 137, Springer, New York, 1992. MR 93f:31001 Zbl 0765.31001

[Baker et al. 1976] G. R. Baker, P. G. Saffman, and J. S. Sheffield, "Structure of a linear array of hollow vortices of finite cross-section”, J. Fluid Mech. 74:3 (1976), 469-476. Zbl 0343.76004

[Barrett and Eremenko 2012] M. Barrett and A. Eremenko, "Generalization of a theorem of Clunie and Hayman”, Proc. Amer. Math. Soc. 140:4 (2012), 1397-1402. MR 2869124 Zbl 1248.32013

[Bell 1992] S. R. Bell, The Cauchy transform, potential theory, and conformal mapping, CRC Press, Boca Raton, FL, 1992. MR 94k:30013

[Brelot 1971] M. Brelot, On topologies and boundaries in potential theory, Lecture Notes in Mathematics 175, Springer, Berlin, 1971. MR 43 \#7654 Zbl 0222.31014

[Carleman 1926] T. Carleman, "Extension d'un théorème de Liouville", Acta Math. 48:3-4 (1926), 363-366. MR 1555232 JFM 52.0316.02

[Castro and Khavinson 2013a] L. de Castro and D. Khavinson, "Analytic functions in Smirnov classes $E^{p}$ with real boundary values", Complex Anal. Oper. Theory 7:1 (2013), 101-106. MR 3010790

[Castro and Khavinson 2013b] L. de Castro and D. Khavinson, "Analytic functions in Smirnov classes $E^{p}$ with real boundary values II”, Anal. Math. Phys. 3:1 (2013), 21-35. MR 3015628 Zbl 06147910

[Crowdy and Green 2011] D. G. Crowdy and C. C. Green, "Analytical solutions for von Kármán streets of hollow vortices", Phys. Fluids 23:12 (2011), Article ID \#126602.

[Davis 1974] P. J. Davis, The Schwarz function and its applications, Carus Mathematical Monographs 17, Mathematical Association of America, Buffalo, NY, 1974. MR 53 \#11031 Zbl 0293.30001

[Duren 1970] P. L. Duren, Theory of $H^{p}$ spaces, Pure and Applied Mathematics 38, Academic Press, New York, 1970. Reprinted by Dover, Mineola, NY, 2000. MR 42 \#3552 Zbl 0215.20203

[Duren et al. 1966] P. L. Duren, H. S. Shapiro, and A. L. Shields, "Singular measures and domains not of Smirnov type”, Duke Math. J. 33 (1966), 247-254. MR 33 \#7506 Zbl 0174.37501

[Ebenfelt et al. 2002] P. Ebenfelt, D. Khavinson, and H. S. Shapiro, "A free boundary problem related to single-layer potentials", Ann. Acad. Sci. Fenn. Math. 27:1 (2002), 21-46. MR 2002m:35227 Zbl 1035.31001

[Fisher 1983] S. D. Fisher, Function theory on planar domains: a second course in complex analysis, Wiley, New York, 1983. Reprinted by Dover, Mineola, NY, 2007. MR 85d:30001 Zbl 0511.30022 
[Friedland and Hayman 1976] S. Friedland and W. K. Hayman, "Eigenvalue inequalities for the Dirichlet problem on spheres and the growth of subharmonic functions", Comment. Math. Helv. 51:2 (1976), 133-161. MR 54 \#568 Zbl 0339.31003

[Gustafsson 1987] B. Gustafsson, "Application of half-order differentials on Riemann surfaces to quadrature identities for arc-length", J. Analyse Math. 49 (1987), 54-89. MR 89b:30032 Zbl 0652.30029

[Hauswirth et al. 2011] L. Hauswirth, F. Hélein, and F. Pacard, "On an overdetermined elliptic problem”, Pacific J. Math. 250:2 (2011), 319-334. MR 2012g:58046 Zbl 1211.35207

[Heins 1950] M. Heins, "A lemma on positive harmonic functions", Ann. of Math. (2) 52 (1950), 568-573. MR 12,259b Zbl 0045.18803

[Hoffman 1962] K. Hoffman, Banach spaces of analytic functions, Prentice-Hall, Englewood Cliffs, NJ, 1962. MR 24 \#A2844 Zbl 0117.34001

[Jones and Smirnov 1999] P. W. Jones and S. K. Smirnov, "On V. I. Smirnov domains", Ann. Acad. Sci. Fenn. Math. 24:1 (1999), 105-108. MR 2000k:30009 Zbl 0921.30004

[Karp 1992] L. Karp, "Construction of quadrature domains in $\mathbb{R}^{n}$ from quadrature domains in $\mathbb{R}^{2}$ ", Complex Variables Theory Appl. 17:3-4 (1992), 179-188. MR 93e:30085 Zbl 0722.30039

[Keldysh and Lavrentiev 1937] M. Keldysh and M. Lavrentiev, "Sur la représentation conformeé des domaines limités par des courbes rectifiables", Ann. Sci. École Norm. Sup. (3) 54 (1937), 1-38. MR 1509363 Zbl 0017.21702

[Kellogg 1929] O. D. Kellogg, Foundations of potential theory, Springer, Berlin, 1929. Reprinted in Grundlehren der Mathematischen Wissenschaften 31, 1967. MR 36 \#5369 Zbl 0053.07301

[Khavinson 1983] D. Khavinson, "Factorization theorems for different classes of analytic functions in multiply connected domains", Pacific J. Math. 108:2 (1983), 295-318. MR 85j:30009 Zbl 0494.30024

[Khavinson 1991] D. Khavinson, "On reflection of harmonic functions in surfaces of revolution", Complex Variables Theory Appl. 17:1-2 (1991), 7-14. MR 92j:31005 Zbl 0702.31004

[Kjellberg 1950] B. Kjellberg, "On the growth of minimal positive harmonic functions in a plane region", Ark. Mat. 1 (1950), 347-351. MR 12,410f Zbl 0040.05502

[Lewis and Vogel 1992] J. L. Lewis and A. Vogel, "On some almost everywhere symmetry theorems", pp. 347-374 in Nonlinear diffusion equations and their equilibrium states (Gregynog, 1989), vol. 3, edited by N. G. Lloyd et al., Progr. Nonlinear Differential Equations Appl. 7, Birkhäuser, Boston, 1992. MR 93j:35078 Zbl 0792.35009

[Markushevich 1977] A. I. Markushevich, Theory of functions of a complex variable, vol. III, English ed., Chelsea, New York, 1977. MR 56 \#3258)

[Pommerenke 1975] C. Pommerenke, Univalent functions, Mathematische Lehrbücher 25, Vandenhoeck \& Ruprecht, Göttingen, 1975. MR 58 \#22526

[Pommerenke 1992] C. Pommerenke, Boundary behaviour of conformal maps, Grundlehren der Mathematischen Wissenschaften 299, Springer, Berlin, 1992. MR 95b:30008 Zbl 0762.30001

[Reichel 1997] W. Reichel, "Radial symmetry for elliptic boundary-value problems on exterior domains", Arch. Rational Mech. Anal. 137:4 (1997), 381-394. MR 98m:35070 Zbl 0891.35006

[Sakai 1981] M. Sakai, "Null quadrature domains", J. Analyse Math. 40 (1981), 144-154. MR 84e: 30069 Zbl 0483.30002

[Shahgholian 1992] H. Shahgholian, "A characterization of the sphere in terms of single-layer potentials", Proc. Amer. Math. Soc. 115:4 (1992), 1167-1168. MR 93c:31011 Zbl 0752.31003 
[Shapiro 1966] H. S. Shapiro, "Remarks concerning domains of Smirnov type", Michigan Math. J. 13 (1966), 341-348. MR 34 \#4472 Zbl 0179.11501

[Shapiro 1992] H. S. Shapiro, The Schwarz function and its generalization to higher dimensions, University of Arkansas Lecture Notes in the Mathematical Sciences 9, Wiley, New York, 1992. MR 93g:30059 Zbl 0784.30036

[Topping 2006] P. Topping, Lectures on the Ricci flow, London Mathematical Society Lecture Note Series 325, Cambridge University Press, 2006. MR 2007h:53105 Zbl 1105.58013

[Traizet 2013] M. Traizet, "Classification of the solutions to an overdetermined problem in the plane", preprint, 2013. arXiv 1301.6927

[Tumarkin and Havinson 1958a] G. C. Tumarkin and S. J. Havinson, "К определению аналитических функций класса $E_{p}$ в многосвязных областях", Uspekhi Mat. Nauk (N.S.) 13:1 (1958), 201-206. MR 20 \#114 Zbl 0087.07902

[Tumarkin and Havinson 1958b] G. C. Tumarkin and S. J. Havinson, “О теореме разложения для аналитических функций класса $E_{p}$ в многосвязных областях”, Uspekhi Mat. Nauk (N.S.) 13:2(80) (1958), 223-228. MR 20 \#3285 Zbl 0081.29804

[Tumarkin and Havinson 1960] G. C. Tumarkin and S. J. Havinson, "Classes of analytic functions on multiply connected domains”, pp. 45-77 in Исследования по современным проблемам теории функций комплексного переменного, Gosudarstv. Izdat. Fiz.-Mat. Lit., Moscow, 1960. In Russian; translated as pp. 37-71 in Fonctions d'une variable complexe: problèmes contemporains, edited by A. I. Markouchevitch, Gauthier-Villars, Paris, 1962. MR 22 \#9616 Zbl 0116.28604

Received May 22, 2012. Revised May 14, 2013.

\section{DMITRY KHAVINSON}

DEPartment OF MATHEMATICS AND STATISTICS

UNIVERSITY OF SOUTH FLORIDA

TAMPA, FL 33620

UNITED STATES

dkhavins@usf.edu

\section{ERIK LUNDBERG}

DEPARTMENT OF MATHEMATICS

Purdue UnIVERSITY

WEST LAFAYETTE, IN 47907

UNITED STATES

elundber@math.purdue.edu

RAZVAN TEODORESCU

Department of MATHEMATICS AND Statistics

UNIVERSITY OF SOUTH FLORIDA

TAMPA, FL 33647

UNITED STATES

razvan@usf.edu 


\title{
PACIFIC JOURNAL OF MATHEMATICS
}

\author{
msp.org/pjm
}

Founded in 1951 by E. F. Beckenbach (1906-1982) and F. Wolf (1904-1989)

\section{EDITORS}

V. S. Varadarajan (Managing Editor)

Department of Mathematics

University of California

Los Angeles, CA 90095-1555

pacific@math.ucla.edu

Paul Balmer

Department of Mathematics

University of California

Los Angeles, CA 90095-1555

balmer@math.ucla.edu

Daryl Cooper

Department of Mathematics

University of California

Santa Barbara, CA 93106-3080 cooper@math.ucsb.edu

Jiang-Hua $\mathrm{Lu}$

Department of Mathematics

Pokfulam Rd., Hong Kong jhlu@maths.hku.hk
The University of Hong Kong

Don Blasius

Department of Mathematics University of California

Los Angeles, CA 90095-1555

blasius@math.ucla.edu

Robert Finn

Department of Mathematics Stanford University

Stanford, CA 94305-2125

finn@math.stanford.edu

Sorin Popa

Department of Mathematics

University of California

Los Angeles, CA 90095-1555 popa@math.ucla.edu

Paul Yang

Department of Mathematics Princeton University

Princeton NJ 08544-1000

yang@math.princeton.edu

\section{PRODUCTION}

Silvio Levy, Scientific Editor, production@msp.org

\section{SUPPORTING INSTITUTIONS}

ACADEMIA SINICA, TAIPEI

CALIFORNIA INST. OF TECHNOLOGY

INST. DE MATEMÁTICA PURA E APLICADA

KEIO UNIVERSITY

MATH. SCIENCES RESEARCH INSTITUTE

NEW MEXICO STATE UNIV.

OREGON STATE UNIV.

\author{
STANFORD UNIVERSITY \\ UNIV. OF BRITISH COLUMBIA \\ UNIV. OF CALIFORNIA, BERKELEY \\ UNIV. OF CALIFORNIA, DAVIS \\ UNIV. OF CALIFORNIA, LOS ANGELES \\ UNIV. OF CALIFORNIA, RIVERSIDE \\ UNIV. OF CALIFORNIA, SAN DIEGO \\ UNIV. OF CALIF., SANTA BARBARA
}

\author{
Vyjayanthi Chari \\ Department of Mathematics \\ University of California \\ Riverside, CA 92521-0135 \\ chari@math.ucr.edu \\ Kefeng Liu \\ Department of Mathematics \\ University of California \\ Los Angeles, CA 90095-1555 \\ liu@math.ucla.edu \\ Jie Qing \\ Department of Mathematics \\ University of California \\ Santa Cruz, CA 95064 \\ qing@cats.ucsc.edu
}

These supporting institutions contribute to the cost of publication of this Journal, but they are not owners or publishers and have no responsibility for its contents or policies.

See inside back cover or msp.org/pjm for submission instructions.

The subscription price for 2013 is US \$400/year for the electronic version, and \$485/year for print and electronic.

Subscriptions, requests for back issues and changes of subscribers address should be sent to Pacific Journal of Mathematics, P.O. Box 4163, Berkeley, CA 94704-0163, U.S.A. The Pacific Journal of Mathematics is indexed by Mathematical Reviews, Zentralblatt MATH, PASCAL CNRS Index, Referativnyi Zhurnal, Current Mathematical Publications and the Science Citation Index.

The Pacific Journal of Mathematics (ISSN 0030-8730) at the University of California, c/o Department of Mathematics, 798 Evans Hall \#3840, Berkeley, CA 94720-3840, is published twelve times a year. Periodical rate postage paid at Berkeley, CA 94704, and additional mailing offices. POSTMASTER: send address changes to Pacific Journal of Mathematics, P.O. Box 4163, Berkeley, CA 94704-0163.

PJM peer review and production are managed by EditFLOW ${ }^{\circledR}$ from Mathematical Sciences Publishers.

\section{PUBLISHED BY}

mathematical sciences publishers

nonprofit scientific publishing

http://msp.org/

(C) 2013 Mathematical Sciences Publishers 


\section{PACIFIC JOURNAL OF MATHEMATICS}

Volume $265 \quad$ No. $1 \quad$ September 2013

Genus-two Goeritz groups of lens spaces

SANGBUM CHO

A compact embedding theorem for generalized Sobolev spaces

SENG-KeE ChUa, SCOTT Rodney and Richard L. WheEden

Partial integrability of almost complex structures and the existence of

solutions for quasilinear Cauchy-Riemann equations

CHONG-KYU HAN and JONG-DO PARK

An overdetermined problem in potential theory

DMITRY KHAVINSON, ERIK LUNDBERG and RAZVAN TEODORESCU

Quasisymmetric homeomorphisms on reducible Carnot groups

XIANGDONG XIE

Capillarity and Archimedes' principle

JOHN MCCUAN and RAY TREINEN

Generalized eigenvalue problems of nonhomogeneous elliptic operators and their application

DUmitru Motreanu and Mieko TANaKa

Weighted Ricci curvature estimates for Hilbert and Funk geometries

SHIN-ICHI OHTA

On generalized weighted Hilbert matrices

EMMANUEL PREISSMANN and OLIVIER LÉVÊQUE

Unique prime decomposition results for factors coming from wreath product 221 groups

J. OWEN SizEMORE and ADAM WinCHESTER

On volume growth of gradient steady Ricci solitons

Guofang Wei and Peng Wu

Classification of moduli spaces of arrangements of nine projective lines

FEI YE 